\title{
Civilisations
}

Revue internationale d'anthropologie et de sciences

humaines

$48 \mid 2001$

La question de l'islam et de l'Etat à l'aube du XXIe siècle

\section{Maroc, légitimité religieuse du pouvoir et démocratie : un couplage impossible}

\section{Ahmed Benani}

\section{(2) OpenEdition}

\section{Journals}

Édition électronique

URL : http://journals.openedition.org/civilisations/3464

DOI : 10.4000/civilisations.3464

ISSN : 2032-0442

Éditeur

Institut de sociologie de l'Université Libre de Bruxelles

\section{Édition imprimée}

Date de publication : 31 août 2001

Pagination : 100-109

ISSN : 0009-8140

\section{Référence électronique}

Ahmed Benani, «Maroc, légitimité religieuse du pouvoir et démocratie : un couplage impossible», Civilisations [En ligne], 48 | 2001, mis en ligne le 01 août 2004, consulté le 30 avril 2019. URL : http:// journals.openedition.org/civilisations/3464; DOI : 10.4000/civilisations.3464 


\title{
MAROC, LÉGITIMITÉ RELIGIEUSE DU POUVOIR ET DÉMOCRATIE : UN COUPLAGE IMPOSSIBLE
}

\author{
Ahmed BENANI
}

\section{INTRODUCTION}

On me permettra d'affirmer une conviction et un constat avant d'entrer dans le vif du sujet : pour que l'option démocratique et läque l'emporte au Maroc, bien des conditions restent à remplir. Cela suppose une conjonction de facteurs de changement qui, à l'heure actuelle, mûrissent de manière inégale. Mais nous pouvons espérer qu'à terme parmi les intellectuels, les femmes, les artistes, les écrivains, les journalistes, les militants politiques, s'affirme un courant de pensée qui ose faire l'éloge de l'individu et mieux encore du citoyen.

Il me paraît impératif de rappeler que le combat dit de libération nationale a promu la langue et la culture arabes, les «valeurs» de l'islam à des rôles de refuge et de repaire, ce qui les a soustrait à l'indispensable critique de la raison.

Les dérives idéologiques à propos de l'arabisation et de l'islam comme modèles d'action historique opposables au modèle «occidental» - en fait à la modernité intellectuelle - ont pris des dimensions alarmantes avec les stratégies de légitimation de l'État-nation (makhzénien ou monarchiste) et la surenchère mimétique des mouvements islamistes qui se présentent comme les vrais héritiers de la légitimité islamique. Ces dérives des imaginaires politiques et sociaux s'opèrent avec l'assentiment, l'engagement sincère ou calculé, de beaucoup d' «intellectuels» très vite devenus «organiques» avec l'État-nation ou opportunistes avecl'islamisme contestataire. Dès lors, il convient de démêler l'écheveau, de clarifier ces notions afin de mieux saisir les enjeux auxquels sont confrontés les sociétés civiles, les citoyens, et de mettre en exergue l'urgence de certaines tâches, dont celle, centrale, de la lutte pour les Droits de l'Homme et la démocratie.

Certes, depuis l'avènement de la révolution islamique iranienne, et plus particulièrement depuis l'émergence des mouvements islamistes, le discours dominant en Occident enferme l'islam ou les islam(s) dans la négativité. Les pratiques religieuses musulmanes sont perçues comme porteuses de conservatisme médiéval et de sectarisme xénophobe. La réponse des islam(s) est, quant à elle, et quelle que soit la diversité de ses acteurs, construite sur l'exacte figure inversée. 
Dans le cadre de cet article, je vais essayer de procéder à un rappel de la légitimité religieuse de la monarchie marocaine et examiner ensuite le statut de l'individu dans ce même pays avant de conclure sur ce que j'ai appelé le couplage impossible si l'on prend en compte le concept de démocratie. Mais pour lever toute ambiguîté ou présupposé idéologique, je souhaite toutefois réaffirmer de manière générale l'existence d'une extrême diversité des sociétés musulmanes, tant au niveau de leurs formes d'expression que de leur développement historique.

Ces sociétés ont toutefois un point commun. Elles sont traversées, de part en part, par les effets déstructurants des luttes politiques, économiques, culturelles qui se déroulent en leur sein et dans le monde. Elles se tournent vers l'islam, qui fonctionne comme modalité de légitimation/ délégitimation des conduites étatiques et/ou sociales, individuelles et collectives, et joue comme instance d'intégration ou de résistance/rejet des forces et des conceptions exogènes. Il en découle une diversité de langages, de stratégies, d'attitudes, d'expressions politiques et culturelles. Ces «expressions de l'islam» concernent non seulement les langages écrits ou parlés, mais également les conduites rituelles, les pratiques économiques, les institutions politiques, l'organisation de l'espace, les créations littéraires, artistiques, techniques, etc..., bref tous les domaines où s'organise l'imaginaire.

Cette référence à l'islam ne date pas d'hier, elle n'est pas imputable en tant que telle aux discours ou à l'activisme des militants de l'islamisme d'aujourd'hui. Il y a sans doute lieu de s'interroger sur le regain des formations discursives à dominante religieuse dans les sociétés musulmanes contemporaines. Mais il est tout aussi impératif de réexaminer le thème du changement social dans une perspective comparatiste et critique avec les deux autres expressions du monothéisme (le christianisme et le judaïsme), et avec les formes de modernité déduites de la rationalité des «lumières» occidentales, mais également du propre champ de la culture islamique. En effet, dans cette tradition plusieurs courants, dès le premier siècle de l'islam, préconisaient l'usage de la raison en matière de connaissance religieuse, fondaient la théologie rationnelle et se prononçaient pour le libre arbitre de l'homme.

\section{LA LÉGITIMITÉ DU MONARQUE MAROCAIN}

\section{Sources et registres}

C'est du positionnement dans le champ religieux que dépend pour la monarchie l'importance de sa légitimité et bien entendu toute réduction ou délégitimation de pouvoir(s) concurrent(s). Plus que tout autre règne, celui de Hassan II, était éminemment sacral. La première question qui vient à l'esprit est celle-ci : par quels chemins la monarchie a-t-elle accédé au statut d'institution sacrée ? De nombreux auteurs ont à ce propos attribué un caractère tridimensionnel à la légitimité du souverain marocain :

- Scripturaire (Coran et Sunna)

- Contractuelle (Bey'a)

- Historique (descendance Chérifienne).

Il s'agit là de trois registres différents, pas nécessairement concordants, qui méritent d'être clarifiés.

La légitimité scripturaire relève de la question du khalifat ou de l'imâmat, comprise ici comme la nécessité religieuse d'un chef (imâm) qui guide la communauté (Umma) et protège le Dar-al-Islam (espace acquis à la Loi Vérité du Coran). Sans rentrer dans une savante digression théologique, on peut avancer que ni le khalifat ni l'imâmat ne peuvent justifier ou 
expliquer l'occupation par la monarchie marocaine de tout le champ politique. D' autant que la version orthodoxe du khalifat ne fait pas du khalife le dépositaire du pouvoir normatif qui n'appartient qu'à Dieu.

La légitimité contractuelle, comme son nom l'indique, s'articule à la notion de contrat et ressort d'un registre plus positiviste qui semble être orienté vers la raison d'État ou de notion d'État de droit.

La légitimité historique enfin est invoquée dans le double sens d'une historicité dynastique et d'une historicité mystique et hagiographique par référence au prophète Muhammad et à sa chaîne symbolique.

\section{Légitimité et constitution}

Dans le milieu des années soixante et compte tenu des très grandes mutations qu'a connues la formation sociale marocaine depuis la fin de la colonisation, une question de fond commençait à préoccuper les sphères dirigeantes de ce pays : la légitimité quasi divine du souverain, lisse et fonctionnant sans aspérité, pouvait-elle suffire à susciter une forme de consensus de la société civile. Pour les sociologues avertis du caractère très composite de la société la réponse était négative, bien que les attitudes puissent varier selon que l'on prenait en compte les acteurs du champ religieux ou les autres, ceux particulièrement engagés dans les formes de luttes politiques à caractère laïque. C'est sur cette toile de fond que la légitimité de type légale-rationnelle (constitutionnaliste) allait intervenir en complément en somme de la précédente. Par rapport à cette nouvelle norme et contrairement au souverain, les élites «modernistes», qui pensent et agissent dans le cadre de l'État-nation, dans lequel selon eux doivent s'enraciner le pluralisme politique et la démocratie, se situent d'entrée de jeu en rupture avec tout dessein de continuité. Par quel cheminement est-on arrivé à cette forme de syncrétisme politique, qui aujourd'hui à son tour, a réactivé les acteurs du contre-champ et leur idéologie islamiste utopiste-messianique?

\section{L'acception hassanienne de la légitimité constitutionnelle}

C'est par la mise en exergue de sa légitimité sacrée que le 18 novembre 1962 Hassan II octroie à son «peuple fidèle» la première constitution du royaume «... Ainsi la Constitution que j'ai construite de mes mains, qui sera diffusée sur tout le territoire du royaume et qui dans un délai de vingt jours sera soumise à ton approbation, cette Constitution est avant tout le renouvellement du pacte sacré, qui a toujours uni le peuple et le Roi».

Cette «Constitution mon bon plaisir» n'est pas «un évènement majeur qui illustre une rupture éclatante avec le passé politique, social, psychologique du Maroc d'antan (...) comme l'écrivait Aveille ${ }^{2}$, qui y voyait «une première consécration officielle de laïcisation d'un régime d'âge théologique ébranlé par les apports techniques et culturels de la civilisation occidentale (...)». Par ironie, on pourrait presque rétorquer à J. Aveille, qu'il s'agit de l'inverse. La référence à la sacralité est, en effet, lourde de sens, elle postule la volonté du Prince de transcender toute hiérarchie des normes et des acteurs politiques. L'objet de la sacralisation apparaît sans confusion aucune : à la fois comme l'institution monarchique et la personne physique du roi. L'équation est en définitive fort simple : choisi par Dieu, le roi ne peut vouloir que le bien de la communauté, toute critique est dès lors impossible et celui qui s'y emploierait fait automatiquement figure d'impie. A ce stade, il est important de rappeler que cette constitution intervient après un long débat ouvert par les représentants du mouvement national dès les accords d'Aix-les-Bains sur le thème de la nécessaire élection au suffrage universel d'une 
assemblée constituante qui aurait pour charge d'élaborer la Loi Fondamentale. Le débat a tourné court, tranché par le monarque qui institua un conseil constitutionnel ${ }^{3}$ coopté et bientôt dissous. Il va de soi que la monarchie allait tout aussitôt jeter aux oubliettes le projet de régime constitutionnel défendu à cette époque et plus tard par le Parti Démocrate pour l'Indépendance (PDI), l'Union Marocaine du Travail (UMT), l'Union des Forces Populaires (UNFP), etc... Les trois constitutions que connaîtra le royaume $(1962,1970,1972)$ seront avant tout des windows dressing des vitrines devant étaler le caractère irréductiblement sacré de la monarchie. Les articles 23 des constitutions de 1970 et 1972 le stipulent sans effet de style : «la personne du Roi est sacrée et inviolable»; quant à l'article 19 de celle de 1972, il précise : «Le Roi, Amir Al Mouminine (Commandeur des Croyants), Représentant Suprême de la Nation, symbole de son unité, garant de la pérennité et de la continuité de l'État, veille au respect de l'Islam et de la Constitution. Il est le protecteur des droits et libertés des citoyens, groupes sociaux et collectivités. Il garantit l'Indépendance de la Nation et l'intégrité territoriale du royaume dans ses frontières authentiques». Ces dispositions ne sont pas qu'incantatoires, elles influent considérablement sur la vie politique du pays et confèrent au roi une posture de déitét . L'argument hagiographique est la clé de voûte de cette sacralité du souverain. Aucune occasion (fête religieuse, réception politique, discours radio-télévisés etc...) n'est négligée pour rappeler l'origine chérifienne du monarque (descendance par filiation agnatique de la fille du Prophète) comme si l'on craignait que sa pertinence historique fusse mise en doute. L'historicité du pouvoir, dont nous parlions, apparaît dans toute sa cohérence et fait l'objet d'une attention particulièrement soignée : la constitution fait de l'obéissance un devoir civique, la sharia en fait une obligation canonique, le Chérifisme la transforme en source de bénédiction.

La constitution, loin de déléguer un quelconque pouvoir aux citoyens, consacre la transcendance et la sacralité du roi; rien ne l'illustre mieux que ce commentaire d'une personnalité très coutumière de Hassan $\mathrm{II}^{5}$ : «Si la souveraineté appartient à la Nation, celleci par la bey'a (l'investiture allégeance, en laquelle peut s'analyser le texte constitutionnel), délègue sa souveraineté au Roi qui en devient seul titulaire et peut, s'il le désire, en subdéléguer partiellement l'exercice en gardant un droit d'évocation qu'il peut faire jouer à tout moment».

Tout l'édifice normatif va se trouver progressivement structuré sous l'impulsion unique du souverain, lequel, par ses initiatives variées que nous verrons ci-après, vise l'abolition ou du moins la réduction de tous les autres centres qui lui disputeraient son monopole du capital symbolique. Avant d'aborder la réaction des acteurs du contre champ religieux et non religieux, il convient pour la clarté de notre propos de préciser l'idée que le roi lui-même se fait de son pouvoir et partant de sa légitimité à partir du texte constitutionnel. Il faut à cet égard dire clairement que c'est avec Hassan II, que naît pour la première fois dans l'histoire du Maroc une véritable philosophie du pouvoir.

Ainsi, dans son adresse célèbre au parlement, Hassan II $^{6}$ donna dans ce sens une véritable leçon de politologie :»Dans son livre sacré, Dieu a dit. «Dis-leur d'agir car votre action sera appréciée par Dieu, par son Prophète et par l'ensemble des croyants», je considère que ce verset a été inspiré à notre Prophète comme s'il avait pour but de nous indiquer la voie à suivre (...). Vous, les élus, vous avez une mission de contrôle. Mais, qui a la charge de contrôler les contrôleurs, c'est Dieu, son Prophète et les croyants. Le contrôle de Dieu, c'est celui de votre conscience. Votre action sera appréciée par Dieu et son Prophète, c'est-à-dire le représentant de son Prophète sur terre qui est le responsable suprême dans le pays. C'est ainsi que se confirme ce que je vous ai toujours affirmé, que vous soyez pouvoir législatif ou pouvoir exécutif, à savoir que si la séparation des pouvoirs est indispensable, elle ne peut en aucun cas concerner la 
responsabilité suprême (...) Et c'est ainsi que nous constatons qu'il résulte du Livre Sacré que tous ceux que Dieu a chargé d'une responsabilité législative ou exécutive doivent obéir à un contrôle : un contrôle de Dieu d'abord, un contrôle de la part de celui que Dieu a chargé des affaires de la communauté musulmane et enfin un contrôle des électeurs «.

\section{L'INDIVIDU AU MAROC ET AU MAGHREB dE MANIĖRE GÉNÉRALE, PROBLĖMES ET PROSPECTIVES}

Si la littérature au sujet de l'individu, l'individualisme et l'humanisme est pléthorique en Occident, l'intérêt pour ces problématiques dans l'espace marocain et maghrébin est fort restreint et en tout cas fort récent. Il est même utile à ce stade de relever que c'est le collectif ou le structurel qui l'emportent. Tous les sociologues ou anthropologues vous le diront, les concepts clés de la sociologie maghrébine restent à ce jour : la zaouïa, le makhzen, la tribu.

Mais que recouvre exactement cette notion d'individu? L'individu existe-t-il dans l'espace arabo-berbère ? Et en définitive qu'est-ce que l'individu : la personne, le sujet libre, le citoyen affranchi ? Quel est le rapport de cet individu à l'autorité parentale, politique, tribale, clanique, etc... Quel est le poids de l'individu face au religieux ? Quels rapports Islam et individu entretiennent-ils?

Encore un mot sur le plan méthodologique, le concept d'individualisme apparaît pour la première fois en France au début du XIX ${ }^{\mathrm{e}}(1826)$, mais la notion dans ce qu'elle a d'intéressant pour nous se pose au moment des États généraux de 1789, où il y a eu un débat portant sur la question de savoir si les individus doivent voter par ordre ou par tête. Autrement dit, s'ils doivent voter en fonction de leur appartenance communautaire aux trois ordres (clergé, tiersétat, noblesse), comme s'ils n'étaient que des représentants de leur communauté, ou bien doivent-ils voter par tête, c'est-à-dire par individu ? Le terme est d'ailleurs utilisé de manière péjorative : il est employé, en fait, pour désigner l'idéologie des Lumières, c'est-à-dire l'idéologie de la table rase - donc déjà une idéologie révolutionnaire -, l'idéologie selon laquelle l'individu pourrait, non seulement faire table rase du passé, mais encore une idéologie selon laquelle l'individu possède une valeur en lui-même, indépendamment de son appartenance communautaire.

Mais revenons au Maghreb et au Maroc en particulier...

La permanence des structures sociales traditionnelles, l'hégémonisme étatique, le jeu des solidarités claniques et familiales, les contraintes de l'économie agraire ou pastorale, imposaient des modes de socialisation des garçons et des filles, des reproductions de «valeurs» et de "représentations» d'autant plus rigides que la pression du système colonial leur conférait la fonction nouvelle de refuge identitaire et de repaire pour éviter les dangers immédiats. D'où encore plus fortement de nos jours la prégnance de l'illusion identitaire.

Les combats dits de libération nationale ont promu la langue et la culture arabes, les «valeurs» de l'islam à ces rôles de refuge et de repaire; ce qui va soustraire précisément ces bastions de la résistance à l'indispensable critique de la raison.

Cette donnée capitale de l'histoire contemporaine du Maghreb n'est pas encore prise en charge par une pensée maghrébine critique; comme nous l'avons déjà souligné, la dérive idéologique à propos de l'arabisation et de l'islam comme modèles d'action historique opposables au modèle «occidental»-en fait à la modernité intellectuelle-a pris des dimensions alarmantes avec les stratégies de légitimation de l'État-nation ou du parti unique et la surenchère mimétique des mouvements islamistes qui se présentent comme les vrais héritiers 
de la légitimité islamique. Pour saisir les enjeux auxquels sont confrontées les sociétés civiles, il apparaît donc nécessaire de clarifier ces notions et de mettre en exergue l'urgence de certaines tâches dont celle, centrale, de la lutte pour les Droits de l'Homme.

Une deuxième réflexion dans la discussion : dans quelle mesure peut-on avoir cette distance critique par rapport à la religion. Car, au fond, l'autorité dans la religion, c'est qu'à un moment ou un autre apparaît l'idée que la loi s'impose aux individus de l'extérieur, c'est-à-dire qu'elle est hétéronome. La question qui pourrait se poser est de savoir si on peut prétendre avoir un rapport critique à la religion, tout en maintenant l'essentiel de la religion, c'est-à-dire ce moment d'autorité indispensable dans la représentation des sources de la loi.

Dans le champ ou le contexte islamique, la pensée qui s'est déployée et exprimée en langue arabe du $\mathrm{VII}^{\mathrm{e}}$ au XII ${ }^{\mathrm{e}}$ siècle, notamment dans l'aire méditerranéenne, a sûrement contribué à l'orientation, au lancement de plusieurs courants de la connaissance à partir du XIII siècle en Europe chrétienne. Mais c'est un fait historique ici encore, que cette pensée qualifiée d'arabe et/ou d'islamique n'a pas pris part à ces explorations nouvelles, à ces découvertes, à ces sauts cognitifs décisifs accomplis en Europe à partir du XVI ${ }^{\mathrm{e}}$ siècle. Bien qu'étroitement lié à cette Europe par les enjeux de la compétition géopolitique en Méditerranée depuis la Reconquista espagnole et l'intervention de l'Empire ottoman, l'ensemble du Maghreb actuel a suivi le destin intellectuel et culturel du monde arabo-islamique et des sociétés africaines : jusqu'au XIX ${ }^{\mathrm{e}}$ siècle, il est resté à l'écart de tout ce qui a constitué et nourri la modernité intellectuelle et scientifique.

Une histoire exacte des premiers éveils à la modernité dans les différents contextes socioculturels maghrébins reste à écrire; on en est resté aux évocations globales et confusionnistes qui s'en tiennent aux grands animateurs de la Nahda ou «Renaissance» arabe, davantage liée au Proche-Orient qu'au Maghreb jusqu'aux années 1940.

On peut dire que l'éveil du Maghreb à une existence historique «moderne» touchant des secteurs étendus de la société s'est effectué grâce aux mouvements nationalistes dont l'audience et la consistance idéologique ont réalisé des progrès décisifs après 1945. Cela veut dire que les idées politiques et juridiques d'émancipation, largement influencées par les postulats de la Raison des Lumières, bien plus que ceux de la Raison islamique, gagnent l'imaginaire social alors que les outils intellectuels de la critique historique, philosophique, juridique, sociologique, psychologique demeuraient - et demeurent encore aujourd'hui - inaccessibles pour l'immense majorité des acteurs sociaux.

\section{Individu, Société et État}

Les problèmes vitaux, les plus anciens et les plus récents, concernant l'histoire, la société, la culture, l'identité du Maghreb demeurent refoulés, escamotés, minimisés, ignorés tandis que gonflent les bavardages soit sur la grandeur méconnue de l'islam et de ses enseignements, les richesses défigurées du patrimoine arabe, la personnalité arabo-islamique du Maghreb longtemps écrasée par le colonialisme, le monde arabe brimé par le sionisme et l'impérialisme euroaméricain..., soit sur l'hostilité radicale et renaissante de «l'Occident» aux tentatives d'émancipation des sociétés arabes et musulmanes.

Cependant, en même temps que se poursuit cette opposition manichéenne entre un araboislamisme salvateur et un Occident cynique, hégémonique et destructeur, tous les acteurs sociaux - industriels, commerçants, politiciens, artistes, chercheurs, ouvriers, paysans valorisent dans leurs conduites pratiques et leurs discours quotidiens - les «valeurs» de la 
modernité soustraites, une fois de plus, à la critique dont elles font l'objet dans leurs lieux de naissance. Mimer maladroitement, ponctuellement, souvent inconsciemment la modernité surtout matérielle et économique, tout en dénonçant idéologiquement l'Occident, est devenu une constante de l'attitude des Maghrébins et plus généralement des Arabes qui demeurent à la fois proches et éloignés des sociétés d'Occident.

Les intellectuels et les chercheurs n'échappent pas à ces tensions et à ces contradictions lorsqu'ils s'emparent de thèmes, de catégories, de définition, d'attitudes de l'esprit constitutifs de la modernité en Occident, pour en rechercher des équivalents, des manifestations, des énoncés en contexte arabe et islamique. C'est le cas pour les droits de l'homme, le droit positif, la démocratie, l'État, la société civile, la laïcité, la sécularisation, l'analyse sociologique, la tolérance, le libéralisme, le socialisme, etc...

Il n'y a pas encore au Maghreb de cadres sociaux de la connaissance comparables à ceux que formait la bourgeoisie des villes européennes aux XVIII ${ }^{e}-\mathrm{XIX}^{\mathrm{e}}$ siècles; $c^{\prime}$ était une classe sociale dynamique, entreprenante sur le planéconomique qui réclamait et traduisait politiquement dans un travail législatif approprié, les idées des Lumières; au Maghreb, les élites politiques qui ont arraché les indépendances se sont hâtées d'étouffer les idées neuves pour imposer une traditionalisation des représentations collectives, des conduites politico-religieuses par un système éducatif régressif et une arabisation islamisation purement tactique et sans exigence scientifique. L'État-nation-parti n'a jamais favorisé l'émergence d'une société civile où l'individu citoyen aurait commencé à faire l'apprentissage de l'autonomie responsable vis-àvis d'un État qui serait alors en relation de réciprocité de droits et de devoirs avec les citoyens dont il devient l'émanation. Cette évolution ne s'est produite dans aucun des régimes maghrébins issus des indépendances; c'est l'autoritarisme de l'État patrimonial qui l'emporte encore.

Les régimes maghrébins par exemple (dès la fin des années 1950, début des années 1960) dans leur processus «légitimatif» se fondent sur des principes réducteurs de l'autonomie individuelle. Les références systématiques à la communauté des croyants, à la collectivité, à la nation, etc..., les autorisent à gérer le destin individuel comme une donnée non séparable du destin collectif. En gros la légitimité politique continue de fonctionner sur des valeurs de groupe englobantes, calamiteuses et mystifiantes; elle exclut le statut de citoyenneté en opérant ou en tentant d'opérer une fusion entre société et appareil d'État ou État-parti.

Le nationalisme, pour les équipes dirigeantes, a été non seulement le creuset obligé de l'intégration sociale mais aussi la panacée à tous les maux de la société.

La question des Droits de l'Homme a été, jusqu'à une date récente, évacuée de l'espace politique maghrébin, non tant à cause du lieu de leur émergence historique (Occident) ou de leur utilisation univoque par ce dernier, qu'en raison de la prégnance d'un ordre social impératif dans ce même espace arabo-berbère. Encore une fois et en schématisant nécessairement, on peut affirmer que les sociétés prises en compte ici concèdent positivement à la violence et à l'autoritarisme, parce que dans la phase pré-islamique elles étaient «anarchiques» et que communautaires ensuite, elles n'ont jamais pu accéderà ce principe inaugurateur d'individualité comme source de légitimité du pouvoir et source de droits opposables à l'État.

On peut donc admettre que la culture politique maghrébine et/ou arabe a généré des pouvoirs despotiques ou du moins une conception patrimoniale de la chose publique. $\mathrm{La}$ configuration actuelle est bien entendu à l'opposé d'un espace de droit et repose sur deux constantes : légitimité imposée et violence. 
L'analyse historique et sociologique de la formation et du fonctionnement des cinq États maghrébins depuis les indépendances montrerait clairement à quel point la notion même d'individu-citoyen a été soit directement ignorée, soit volontairement escamotée dans la législation, la pratique administrative, le système éducatif, la vie culturelle, les mécanismes de contrôle idéologique. Si l'on excepte les premières conquêtes de la condition féminine en Tunisie grâce à Bourguiba, le statut juridique des femmes et de l'enfant, après quarante ans d'indépendance, suffit à établir le poids de la vision patriarcale et de la traditionalisation dans la «culture» des «élites» politiques qui ont contrôlé les appareils étatiques. On peut aussi citer les conflits récents et toujours ouverts entre les États et les «citoyens» au sujet des ligues des droits de l'homme : officiellement, on adopte avec détermination tout le discours occidental sur les droits de l'homme; dans la pratique, on exerce un contrôle étroit sur les animateurs des ligues, leur travail, leurs relations avec l'extérieur.

Le plus important, cependant, dans les sociétés maghrébines contemporaines, c'est l'absence d'un débat démocratique continu, libre, ouvert aux grands problèmes internes à chaque société aussi bien qu'aux défis changeants de la vie internationale, de l'économie mondiale, de l'ordre - ou désordre - monétaire, de la philosophie libérale, des stratégies géopolitiques. Les hommes et les femmes capables de nourrir ce débat ne manquent pas; mais il n'y a ni les cadres sociaux qui le transformeraient en un mouvement politique ou intellectuel significatif, ni des espaces institutionnels qui l'amplifieraient tout en garantissant la pertinence. On retrouve la question de l'individu-citoyen qui cherche à émerger, mais qui ne trouve pas de points d'appuis solides ni dans la société, ni dans les diverses sphères de l'expression du pouvoir, ni dans une culture arabo-islamique revendiquée comme l'outil essentiel de réalisation de soi, mais encore silencieuse sur les tensions éducatives de la modernité.

\section{Individu et langue}

Ainsi, les grands axes d'une réflexion poussée pour l'élaboration d'une pensée maghrébine restent encore à identifier, à circonscrire, à conduire en relation avec les impatiences d'une population extrêmement jeune. Je parle d'une pensée, car elle est encore à naître, à s'affirmer, à s'imposer comme telle; la pensée maghrébine n'existe pas, non pas comme le répètent toujours les idéologies nationalistes parce que le colonialisme en a empêché l'exercice, mais parce que la situation linguistique du Maghreb depuis les Romains a toujours été caractérisée par une langue savante écrite maîtrisée par une minorité, et des dialectes locaux oraux, arabes ou berbères, que les élites, dans toutes les phases de l'histoire, n'ont jamais assumé pour promouvoir l'un d'eux au rang de langue «nationale». Les penseurs, les écrivains, les savants se sont toujours exprimés dans une langue et un niveau de cette langue qui les projettent dans un espace culturel extra maghrébin.

Cela est vrai encore de nos jours, malgré les progrès réels de l'arabe standard qui reste éloigné de l'arabe classique des grands savants et penseurs de l'époque médiévale, aussi bien que des appareils conceptuels en perpétuelle évolution dans tous les domaines de la recherche scientifique dans le monde. L'isolement intellectuel de l'arabe standard, dominé par les usages idéologiques, mythologiques, a des conséquences particulièrement visibles dans le domaine des sciences de l'homme et de la société qui, seules, par leurs démarches critiques, pouvaient compenser, atténuer les ravages causés par une arabisation à finalité politique, indifférente justement aux faiblesses anciennes et nouvelles de la pensée maghrébine.

L'identification d'une pensée maghrébine est une tâche passée sous silence, d'autant plus qu'une réflexion sur sa mise en œuvre impliquerait aux yeux des 
tenants de l'orthodoxie, une atteinte au dogme idéologique du Maghreb arabe indissociable de la Nation arabe ou de l'islam orthodoxe.

Ce n'est pas ici le lieu d'énumérer ces tâches qui permettraient, si elles sont remplies correctement, l'émergence intellectuelle et culturelle de l'individu-citoyen au Maghreb. Disons ici quelques mots, cependant, sur la plus lourde, la plus urgente, la plus difficile et, en même temps, la plus refoulée jusqu'ici : c'est la question de l'islam abordée sous l'angle de l'anthropologie culturelle, de l'histoire comparée des religions, de l'histoire des systèmes de pensée théologique et philosophique.

\section{Individu et religion}

C'est ici que se révèle le plus clairement la contradiction fondamentale de l'histoire du Maghreb depuis sa conversion à l'islam malékite qui, après le départ des Fatimides en 969, s'est imposé sans partage (sauf pendant l'intermède ottoman où le hanafisme a gagné quelques partisans en Tunisie et en Algérie) jusqu'à nos jours. Non seulement le pluralisme de la pensée islamique ne s'est guère imposé au Maghreb, mais les questions fondamentales débattues en théologie, en philosophie, en méthodologie et principiologie du droit, en exégèse, en mystique ont été oubliées, refoulées depuis la disparition d'Ibn Rushd (de Shâtibi, d'lbn Sub'in, d'Ibn 'Arafa)

La question de l'islam ressurgit en plein $\mathrm{XX}^{\mathrm{e}}$ siècle comme un refuge identitaire et un levier de la lutte nationaliste. Les réformistes comme Ben Badis, Tahar Ben Achour, Allal al-Fàsi révèlent davantage l'impensé accumulé dans la perception maghrébine de l'islam que la prise de conscience des tâches réelles rendues pourtant plus évidentes par l'occupation coloniale. Le rétrécissement du champ intellectuel de la pensée islamique s'est paradoxalement accéléré durant les années d'indépendance, alors que dans la phase des luttes de libération, on pouvait entrevoir des possibilités d'une sortie de la religion, comme l'Europe l'avait fait vis-à-vis du christianisme. On était loin, en tout cas, de la radicalisation fondamentaliste qui domine aujourd'hui dans un grand nombre de sociétés musulmanes.

Ici encore, les historiens des années 1960-92 devront expliquer les processus et les forces qui ont conduit d'un socialisme arabe d'essence nettement laïque à un fondamentalisme inédit dans toute l'histoire de la pensée islamique. Parmi les facteurs qui expliquent cette évolution, on retiendra l'ignorance généralisée au Maghreb, bien avant la phase coloniale, de la dimension intellectuelle pluraliste de la pensée islamique. Plus gravement encore, le système éducatif mis en place par les États nationalistes a encouragé un enseignement obscurantiste de la religion, inconciliable même avec les positions développées par les penseurs médiévaux. A ce jour, l'enseignement de l'histoire comparée des religions, de l'anthropologie sociale et culturelle, les techniques de l'exégèse des textes sacrés, des différents systèmes théologiques dans les trois religions monothéistes, reste pratiquement inexistant dans les universités du Maghreb et, plus généralement, du monde musulman. On a, en revanche, renforcé ou conservé les enseignements traditionnels des sciences islamiques dans les universités anciennes (Zitouna, Qurawiyyin) ou nouvelles (Constantine, Dâr al-Hadith al-Hasaniyya). Ce n'est sûrement pas là que l'islam peut être étudié et interprété comme une des manifestations historiques du fait religieux présent dans toutes les sociétés.

Ainsi, les sociétés maghrébines consomment à outrance du religieux, du sacré, du surnaturel, du divin, du prophétisme... sans possibilité de recours à des instances intellectuelles et scientifiques qui éclaireraient la complexité des phénomènes, des croyances, des conduites, 
des représentations liés à chacun de ces grands concepts. Les grands débats introduits en Europe par des penseurs chercheurs comme Émile Durkheim ou Max Weber, ou même, dès les XVII ${ }^{e_{-}}$ $\mathrm{XIX}^{\mathrm{e}}$ siècles, par Spinoza, Kant, Fichte, Hegel, Marx...sur la religion n' ont encore touché, au Maghreb, que quelques professeurs et étudiants isolés. Les uns subissent le poids grandissant d'un discours religieux dévoyé par le militantisme politique, les autres exploitent avec jubilation le désarroi des jeunes sans travail, sans ressources, sans horizons de délivrance en dehors du salut.

Ne laissons place à aucun malentendu : l'étude moderne de la question religieuse n'a rien à voir avec l'attitude réformiste qui prône un retour aux sources authentiques et aux enseignements «vrais» de l'islam originel; l' anthropologie enseigne que cette attitude est d'essence mythologique puisqu'elle enracine la vérité totale, intangible dans un temps inaugurateur. Ce que le Maghreb et toute la pensée islamique doivent réussir, c'est une sortie du religieux non pas par l'abandon et le discrédit jeté sur les religions comme l'a fait un certain laïcisme militant en France, mais par la conquête d'une distance critique, raisonnée à l'égard des manifestations multiples et complexes du religieux dans les sociétés.

On voit bien que le parcours intellectuel et culturel suggéré ici est un des préalables à l'émergence, à l'existence active et féconde de l'individu-citoyen-personne au Maghreb. La démocratie, les droits de l'homme, l'épanouissement de la personne en tant qu'individu autonome et citoyen responsable, restent des mots creux, des évocations gratuites si une culture libératrice, ouverte, fondée sur l'interrogation infinie, la suspicion éducative, la critique continue des «valeurs», n'est pas prodiguée à tous les citoyens.

Il faut donc penser et agir dès lors en tenant compte de tout ce qui sépare les Maghrébins des conquêtes de la modernité. Ils vivent pourtant depuis des siècles sur les rivages d'une mer où ont circulé les idées, les découvertes, les techniques, les croyances qui ont préparé et nourri cette modernité. Le Maghreb retrouvera-t-il les fragments de son identité éclatée, renouera-til les liens de sa véritable histoire ou continuera-t-il à cheminer vers un destin qui n'est pas le sien, mû par des montages phantasmatiques sur ses histoires «nationales», sa religion, sa personnalité, sa vocation... Ce qui se passe en Algérie, depuis octobre 1988, illustre tragiquement ce parcours, tout en laissant espérer à tous les Maghrébins une meilleure maîtrise de leur histoire présente. A condition que soit enfin assurée à tous la liberté de penser, d'écrire, de publier, de critiquer, de croire ou de ne pas croire à des systèmes produits et contrôlés par des agents sociaux. Libertés élémentaires et, pourtant, neuves et toujours espérées, réclamées par des Maghrébins qui ont consenti assez de sacrifices pour en jouir désormais pleinement.

\section{CONCLUSION : QUEL BILAN, QUELLES PERSPECTIVES ESQUISSER DĖS LORS ?}

Au Maghreb il y a toujours eu recherche d'un espace d'identification globale, d'un lieu de rassemblement symbolique; jusqu'à la colonisation, il y aura eu deux cercles d'allégeance celui de l'identité lignagère ou ethnique et celui de l'identité religieuse. Depuis l'indépendance ou un peu avant, il y aura comme en surimpression, un troisième cercle - celui de l'identité nationale - qui, à l'impulsion des intellectuels ou des lettrés de la classe moyenne ou bourgeoise, se dégage peu à peu de l'étreinte des deux premiers, en prenant pour levier la sécularisation de la vie politique. Mais les éléments de cristallisation d'une personnalité proprement nationale ne sont pas donnés à l'avance. Cette entité nationale est indécise, elle oscille constamment entre des repères ethniques et religieux, linguistiques et politiques instables. Ainsi, par exemple, la population de chacun des pays arabes continuera-t-elle longtemps d'hésiter entre plusieurs appartenances enchevêtrées : l'entité politique qui la 
constitue en État, l'aire culturelle qu'elle partage avec le reste du monde arabe et la communauté islamique à laquelle elle adhère sentimentalement.

Pour que l'option démocratique et laïque l'emporte, bien des conditions restent à remplir. Cela suppose une conjonction de facteurs de changement qui, à l'heure actuelle, mûrissent de manière inégale; j'en citerai trois d'ordre différent.

1. Un facteur idéologique-culturel : il faut que, parmi les intellectuels, les femmes, les artistes, les écrivains, les journalistes, les militants politiques, s'affirme un courant de pensée qui ose faire l'éloge de l'individu.

2. Un facteur socio-économique : il faut que le rôle social rempli par l'individu déborde le cadre du travail intellectuel, du fonctionnariat, etc... pour occuper un créneau décisif dans l'activité de production. On le sait, il y a une différence fondamentale entre l'Europe des Lumières et le Sud d'aujourd'hui. Dans ce dernier, il n'y a pas de force sociale productive et dynamique, susceptible d'offrir à la démocratie une assise économique viable. La question posée est celle de savoir si un capitalisme moderne et social peut, à l'heure de la mondialisation et de ses terribles contraintes, voir le jour ou si le Sud doit, aux côtés d'un secteur économique d'État sous contrôle démocratique, inventer des formes de production et de distribution qui soient, à la fois non étatiques et non capitalistes.

3. Enfin et tertio, un facteur international : il faut que l'environnement mondial permette ces mutations intérieures. Le Sud ne pourra pas se payer l'équivalent d'une révolution industrielle européenne. D'abord parce que le Nord domine le marché mondial et ne le permettra pas; ensuite parce que les moyens de communication actuels créent une conscience mondiale de ce qui est tolérable et de ce qui ne l'est pas. Or les sacrifices demandés aux classes laborieuses européennes au XIX ${ }^{\mathrm{e}}$ siècle et dans la première moitié du XX $\mathrm{X}^{\mathrm{c}}$ ne pourront pas être exigés des classes laborieuses du Sud. Celles-ci formulent des besoins et des exigences désormais conformes à des normes universelles.

\section{NOTES}

1 Hassan II, discours du 18 novembre 1962 : «Le Maroc en marche», Rabat, Ministère de l'Information, 1965, p. 198.

2 Aveille (J.) «Le Maroc se donne une monarchie constitutionnelle», Confluent $\mathrm{N}^{\circ} 27$ Janvier 1963, pp. 6-7.

3 Dahir (décret) du 3 novembre 1960, Bulletin Officiel du 4 novembre 1960, p. 1898.

4 Le roi est à l'abri de toute atteinte physique et morale et de toute poursuite judiciaire, et toute atteinte à cette intégrité est passible de peines et de sanctions dont les modalités sont consignées dans le code pénal (articles 163 à 186 du code pénal marocain),il ne peut être l'objet de critique ni être représenté d'une manière humoristique». L'article 37 de la constitution de 1972 déclare «Aucun membre de la Chambre des représentants ne peut être poursuivi ou recherché, arrêté, détenu ou jugé à l'occasion des opinions ou votes émis par lui dans l'exercice de ses fonctions, hormis le cas où les opinions exprimées mettent en cause le régime monarchique, la religion musulmane, ou constituent une atteinte au respect dû au roi».

5 Ahmed ALAOUI, ministre d'État et président du trust de la presse gouvernementale Le Matin du Sahara et «Le Soir», dans son éditorial «Du pouvoir constituant». Le Matin du Sahara du 28 février 1972, soit quelques jours avant l'organisation du référendum constitutionnel de 1972.

6 Hassan II, discours du 13 novembre 1978, définissant les pouvoirs du Parlement, in Le Matin du Sahara du 16 octobre 1978. 